\title{
Necroptosis of infiltrated macrophages drives Yersinia pestis dispersal within buboes
}

\author{
Mohammad Arifuzzaman, ${ }^{1}$ W.X. Gladys Ang, ${ }^{1}$ Hae Woong Choi, ${ }^{2}$ Matthew L. Nilles, ${ }^{3}$ \\ Ashley L. St. John, ${ }^{2,4}$ and Soman N. Abraham ${ }^{1,2,4,5}$ \\ 'Department of Molecular Genetics and Microbiology and 'Department of Pathology, Duke University, Durham, North \\ Carolina, USA. ${ }^{3}$ Department of Biomedical Sciences, School of Medicine and Health Sciences, University of North Dakota, \\ Grand Forks, North Dakota, USA. ${ }^{4}$ Program in Emerging Infectious Diseases, Duke-National University of Singapore, \\ Singapore, Singapore. ${ }^{5}$ Department of Immunology, Duke University, Durham, North Carolina, USA
}

\begin{abstract}
When draining lymph nodes become infected by Yersinia pestis (Y. pestis), a massive influx of phagocytic cells occurs, resulting in distended and necrotic structures known as buboes. The bubonic stage of the $Y$. pestis life cycle precedes septicemia, which is facilitated by trafficking of infected mononuclear phagocytes through these buboes. However, how $Y$. pestis convert these immunocytes recruited by host to contain the pathogen into vehicles for bacterial dispersal and the role of immune cell death in this context are unknown. We show that the lymphatic spread requires Yersinia outer protein J (YopJ), which triggers death of infected macrophages by downregulating a suppressor of receptor-interacting protein kinase 1-mediated (RIPK1-mediated) cell death programs. The YopJ-triggered cell death was identified as necroptotic, which released intracellular bacteria, allowing them to infect new neighboring cell targets. Dying macrophages also produced chemotactic sphingosine 1-phosphate, enhancing cell-to-cell contact, further promoting infection. This necroptosis-driven expansion of infected macrophages in buboes maximized the number of bacteria-bearing macrophages reaching secondary lymph nodes, leading to sepsis. In support, necrostatins confined bacteria within macrophages and protected mice from lethal infection. These findings define necrotization of buboes as a mechanism for bacterial spread and a potential target for therapeutic intervention.
\end{abstract}

License: This work is licensed under the Creative Commons Attribution 4.0 International License. To view a copy of this license, visit http://creativecommons.org/ licenses/by/4.0/.

Conflict of interest: SNA is the cofounder and chief scientific officer for Mastezellen Bio Inc

Submitted: May 14, 2018 Accepted: August 7, 2018 Published: September 20, 2018

\section{Reference information:} JCI Insight. 2018;3(18):e122188. https://doi.org/10.1172/jci. insight.122188.

\section{Introduction}

Draining lymph nodes (DLNs) are organized structures of immune cells that constantly survey the lymph for pathogens (1). Many pathogens, such as Yersinia pestis ( $Y$. pestis), are lymphotropic and rely on the lymphatic system as a conduit to reach a systemic infection (2-4). Y. pestis is the etiological agent of bubonic plague, a disease responsible for 28 million deaths in the 14th century and that remains a significant infectious threat today $(5,6)$. It is best known for eliciting the formation of prominent buboes following dermal inoculation of the pathogen by fleas (5). $Y$. pestis infection results in a specific pathology of DLNs, where the normally quiescent structures become massively swollen, containing unusual infiltrations of myeloid cells, tissue necrosis, and a substantial burden of intra- and extracellular bacteria. These inflamed DLNs grow to more than twice the size of a regular hypertrophic lymph node and no longer retain their native architecture (7). It could be assumed that immune cell infiltration would be beneficial for pathogen clearance in DLNs; however, during $Y$. pestis infection, infiltrating cells are frequent targets of infection. Recent studies suggest that $Y$. pestis spread initially via the lymph by hitchhiking within mononuclear phagocytes that traffic from node to node and finally entering the blood circulation (4).

Interestingly, a striking but largely overlooked feature of $Y$. pestis-infected buboes is the extensive necrosis seen throughout this organ (8). Indeed, biopsies of necrotic regions of buboes reveal mostly cell debris and bacteria, suggesting cell lytic events (7). Although first described a century ago, the precise role of this cell death during $Y$. pestis infection is unknown. Since the necrosis in buboes appears to precede systemic infection (4), we questioned if this cytolytic activity contributes to bacterial septicemia. In vitro studies have 
demonstrated that programmed cell death can be triggered in macrophages by $Y$. pestis infection. This was attributed to a bacterial factor Yersinia outer protein J (YopJ), an acetyl transferase produced by Y. pestis and related Yersinia species (9-11). The host molecular components of this programmed cell death include caspase 8 and receptor-interacting protein kinase 1 (RIPK1) (12). For Y. pestis, the role of YopJ as a virulence factor is less clear. While it has been shown to inhibit innate immune NF-кb signaling, antagonize TNF production, and induce apoptosis of immune cells, it has also been reported to activate inflammosomes, which are powerful recruiters of pathogen-clearing immune cells $(11,13)$. We began our study with a proposition that a highly effective pathogen such as $Y$. pestis may have successfully coevolved with the host to encode virulence factors that are beneficial for bacterial infection and spread. We also aimed to further characterize the types of cell death induced by $Y$. pestis, since, recently, our understanding of nonapoptotic programmed cell death has expanded (14). Necroptosis, a form of regulated necrosis, has been shown to be induced by various pathogen-associated molecular patterns (PAMPs) but also negatively regulated by prosurvival factors at various steps of the signaling pathway (15). Interestingly, the necroptotic pathway is tightly linked with apoptosis, and therefore, a given death stimulus may lead to either form of cell death, and inhibition of one form may lead to activation of the other (15-17), blurring interpretation of cell death programs involved. However, the recent discoveries of the necrosome as an integral necroptosis-inducing signaling complex and the pore-forming protein mixed-lineage kinase domain-like (MLKL) as the executor of necroptosis have now provided specific molecular markers to pinpoint necroptosis $(18,19)$.

Here, we describe that YopJ-triggered necroptosis is consequential to the spread and escape of $Y$. pestis from buboes. Targeting immune cells and triggering their death is a way to not merely suppress antimicrobial activities, but rather a systematic expansion of intracellular infection. Unlike non-bubo-forming infections by other Yersinia species, when this pathway is triggered in Y. pestis-infected buboes, it has the effect of allowing bacterial release from immune cells and massively amplifying the numbers of bacteria within buboes and their potential to infect new cellular targets. Furthermore, we identified that the signaling program induced by $Y$. pestis within dying cells involves S1P production, which brings new uninfected cellular targets proximal to the necroptotic host cells, further augmenting infection. This potentially novel mechanism of bacterial spread explains how $Y$. pestis exploits the host immune response that is generated in the lymph node to achieve successful infection.

\section{Results}

YopJ is a critical virulence factor promoting bacterial dispersal. To address the question of whether YopJ influences bacterial dissemination through buboes, we undertook a mouse challenge study where the pathogen was inoculated into rear footpads to mimic the natural intradermal route of infection. This site is drained by a single lymph node, the popliteal node (PN), which in turn is drained by the iliac nodes (INs) (Supplemental Figure 1A; supplemental material available online with this article; https://doi.org/10.1172/ jci.insight.122188DS1). We initially confirmed that footpad infection of mice with $Y$. pestis led to bubo formation (characterized by massive influx of CD11b+ leukocytes in the DLN) and infection of the node, followed by septicemia (Supplemental Figure 1, B and C), consistent with prior studies $(4,7)$. When we instilled a lethal dose of WT $Y$. pestis Kim5 strain or a comparable bacterial dose of the isogenic $\Delta y o p J$ mutant, most of the Kim5-infected mice died by day 7, whereas all mice infected with the $\Delta y o p J$ strain survived (Figure 1A). The survival rate of mice infected with a $\Delta y o p J$ strain complemented with yopJ encoded in a low-copy expression vector was comparable with that of mice infected with the WT Kim5 strain (Figure 1A), confirming that YopJ is a potent virulence factor promoting the pathogenesis of $Y$. pestis.

To examine if the YopJ-mediated lethality coincided with enhanced bacterial dissemination, we compared bacterial numbers in the blood 48 hours postinfection (h.p.i.), which is when bacterial infection becomes systemic (Supplemental Figure 1C). We found that the numbers of $\Delta y o p J$ mutant bacteria were significantly lower than Kim5 bacteria in the blood (Figure 1B). A similar difference in bacterial numbers was also observed in the spleen 72 h.p.i. (Figure 1C), suggesting that YopJ promotes bacterial dissemination. Next, we compared bacterial numbers in the PNs and INs, the primary and secondary lymph nodes encountered early in the infectious process, respectively. We found that by 24 h.p.i., both Kim 5 and $\Delta y o p J$ bacteria induced the formation of large buboes (Supplemental Figure 1D). Consistent with our data from spleen and blood, we observed a significant difference between Kim5 and $\Delta y o p J$ bacteria in the INs (Figure 1D), but remarkably, we did not observe any such difference between the 2 strains in the PNs (Figure 1E). This suggested that $Y$. pestis lacking YopJ have no defects in entry into the lymphatic system, but subsequent stages of infection within or beyond the primary lymph node are affected. 
A

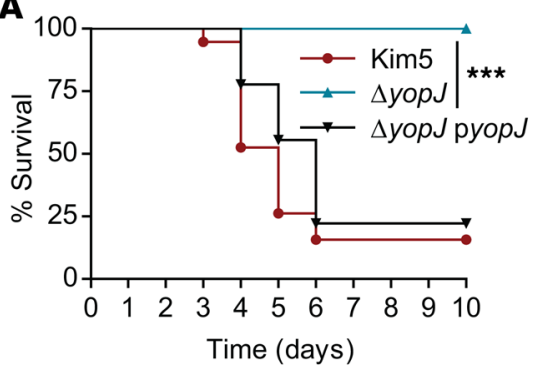

B

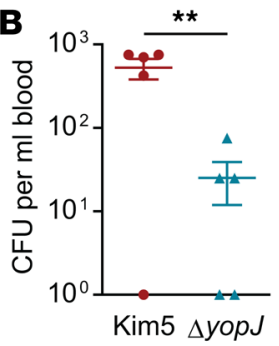

E

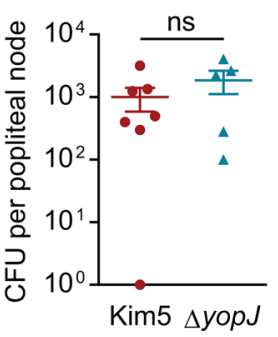

F

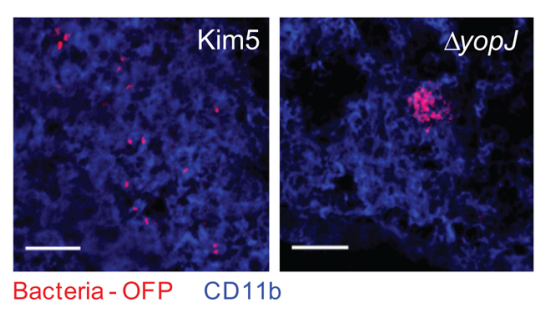

C
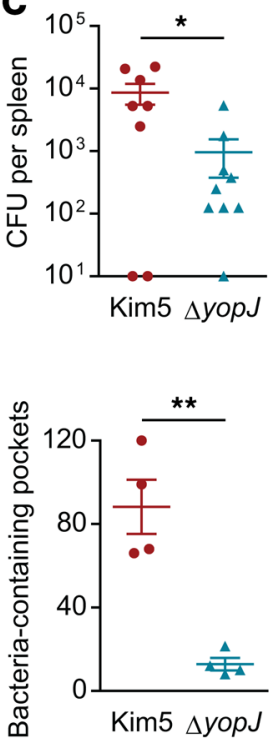

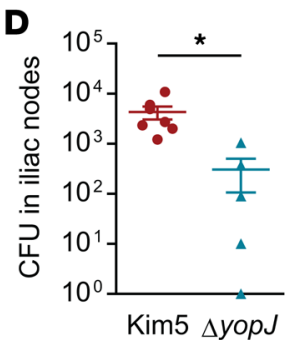

G

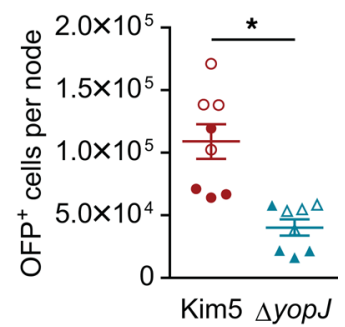

Figure 1. YopJ is a critical virulence factor promoting bacterial dispersal. (A) Survival of mice challenged with bacteria instilled into a single rear footpad. Data are combined from 2 independent experiments, $n=9-10$. (B) Bacterial numbers (CFU) in the blood, 48 hours after footpad infection with Kim5 or $\Delta$ yopJ strain $(n=5)$. (C) Bacterial numbers in spleen, 72 hours after infection (h.p.i.) ( $n=8-9)$. (D and E) Bacterial numbers in iliac nodes (INs) (D) and popliteal nodes (PNs) (E) 24 h.p.i. ( $n=5-7)$. Data are representative of 3 independent experiments. (F) Immunofluorescence staining of PN cross-sections 24 hours after footpad infection with OFP-labeled Kim5 or $\Delta y o p /$ bacteria. The images are representative of 2 independent experiments. Scale bar: $25 \mu \mathrm{m}$. The graph shows bacteria-containing pockets counted in 4 fields $(n=4)(\mathbf{C})$ Quantification of OFP+ cells in PNs 24 h.p.i. by flow cytometry. Data are combined from 2 independent experiments (distinguished by open and closed symbols), each with 4 mice per group. Significance for Kaplan-Meier curves was determined by the log-rank test. Other data were analyzed via unpaired 2-tailed Student's $t$ test. ${ }^{*} P<0.05,{ }^{* *} P<0.01,{ }^{* * *} P<0.001$.

To investigate bacterial distribution and compartmentalization within nodes further, we infected mice with orange fluorescent protein-labeled (OFP-labeled) bacteria and then examined cross-sections of the primary PN 24 h.p.i. for the bacilli. Knowing that phagocytic cells, particularly of the monocyte/macrophage lineages, are the primary targets for $Y$. pestis infection and that they aid in the trafficking of bacteria between nodes, we examined whether $Y$. pestis could infect those cells efficiently in the absence of YopJ. Surprisingly, although both Kim5 and $\Delta y o p J$ strains appeared to be within $C D 11 b^{+}$phagocytic cells, $\Delta y o p J$ bacteria were found to be restricted in large pockets that were relatively few in number. In contrast, Kim5 Y. pestis appeared well dispersed throughout the node (Figure 1F), suggesting YopJ is important for bacterial spread within a bubo. Indeed, flow cytometry of single cell suspensions from PNs confirmed that the numbers of bacteria-harboring cells in Kim5-infected buboes were significantly higher than in $\Delta y$ opJ-infected buboes (Figure 1G), even though the total numbers of bacteria in Kim5- and $\Delta y o p J$-infected PNs were comparable (Figure 1E). These data show that YopJ is a potent virulence factor that promotes bacterial dissemination in the primary DLN.

YopJ-triggered macrophage death promotes infection of neighboring cells. Since we noted significantly higher numbers of Kim5-bearing macrophages, compared with the $\Delta y o p J$-bearing macrophages within the PNs (Figure 1, F and G), we hypothesized that YopJ-dependent cell death initiated bacterial spread within the DLN. Major phagocytic cells infiltrated into buboes at 24 h.p.i. were macrophages, monocytes, and neutrophils (Supplemental Figure 2, A and B), consistent with previous reports $(4,7)$. Based on the role of monocytes and macrophages in $Y$. pestis trafficking between nodes (4) and on our observations here that they are the major targets of Y. pestis (Supplemental Figure 2, C and D), we utilized J774A.1 cells, which display a similar phenotype to the target cell population in buboes, as an in vitro model system to test whether YopJ-dependent cell death could trigger bacterial release from cells. Cells were infected with OFP-labeled $Y$. pestis at an MOI of 10:1, exposed to gentamycin to kill extracellular bacteria, and transferred onto a monolayer of uninfected macrophages at a ratio of 1:10 (infected/uninfected). The dissemination of infection was then tracked by time-lapse fluorescence microscopy. Upon addition of Kim5-OFP-infected cells onto uninfected cells, we observed exteriorization of bacteria from the infected cells, followed by infection of multiple neighboring cells (Figure 2A, top panels, and Supplemental Video 1). In contrast, addition of cells bearing $\triangle y o p J-O F P$ bacteria did not result in infection of neighboring cells (Figure 2A, bottom panels, 
A
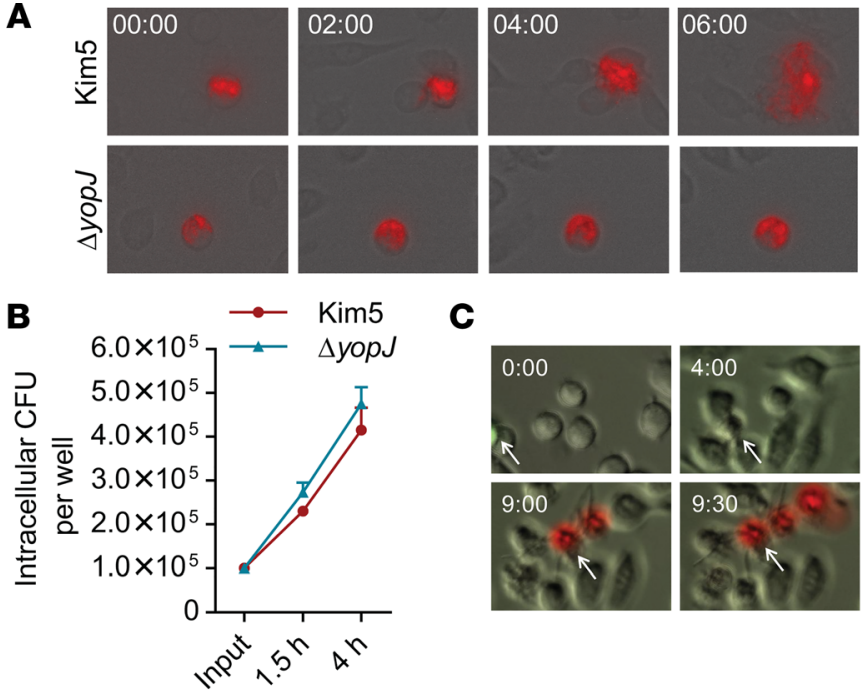

C
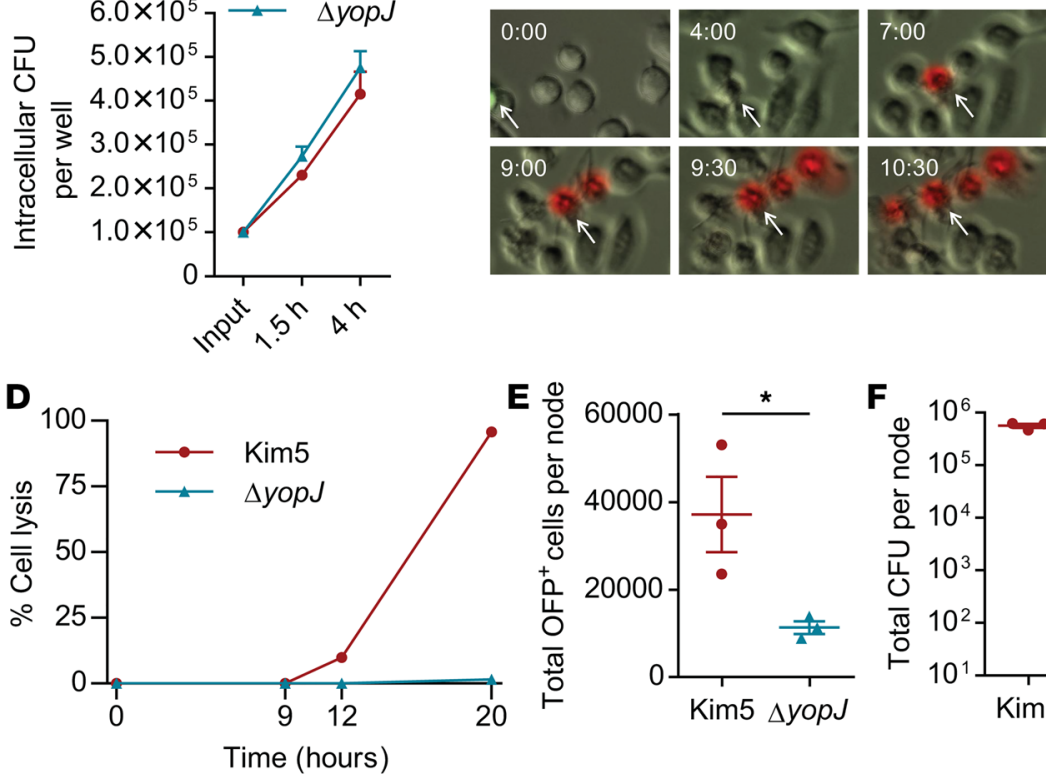

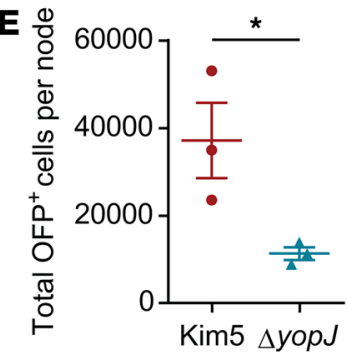

Figure 2. YopJ-triggered macrophage death promotes infection of neighboring cells. (A) Time-lapse images showing intracellular OFP+ bacteria (red) and their release from J774A.1 macrophages (magnification 200×). Time 00:00 (hh:mm) indicates 4 hours after initial infection. Also see Supplemental Videos 1 and 2 . (B) Number of intracellular bacteria per well at 1.5 hours and 4 h.p.i. of $2 \times 10^{4} \mathrm{J774A} .1$ cells for $30 \mathrm{~min}$. $(n=3)$. Input indicates CFU count at 0 min. from the bacterial suspension added to each well. (C) Cytolysis visualized by the entry of propidium iodide (red) into J774A.1 macrophages (magnification 200x). Time 00:00 (hh:mm) indicates 4 hours after initial infection. The arrow in each panel indicates the initially infected cell. Ratio of infected/uninfected cells, 1:10. Also see Supplemental Video 3. (D) Percent cell lysis representing death of initially and newly infected cells combined at various time points after initial infection. Ratio of initially infected/uninfected cells, 1:10. Data are represented as the mean of duplicate for each time point. (E and F) Total OFP+ cells (E) and bacterial count (F) in PNs 9 hours after footpad injection with J774.1A cells bearing Kim5-OFP or $\triangle$ yopJ-OFP $(n=3)$. Data are representative of at least 2 independent experiments. Data were analyzed via unpaired 2-tailed Student's $t$ test. ${ }^{*} P<0.05$.

and Supplemental Video 2). Indeed, these yopJ-OFP bacteria could not escape their host cell, even though they multiplied intracellularly at a similar rate to Kim5-OFP (Figure 2B). These data suggest a contribution of YopJ to bacterial escape from infected macrophages.

If $Y$. pestis were to exploit macrophages as a reservoir followed by a burst of bacterial release due to cell death, we expect a close association between cytolysis and bacterial exteriorization. To visualize cytolysis during infection, we labeled macrophages with a green fluorescent dye and infected them with Kim5 bacteria, followed by gentamycin treatment, to kill extracellular bacteria. These labeled and infected macrophages were then placed on a monolayer of unlabeled and uninfected macrophages. Propidium iodide was added to the extracellular medium so that cells losing membrane integrity could be readily visualized by time-lapse microscopy. We observed that lysis of an originally infected macrophage was followed sequentially by lysis of neighboring macrophages (Figure 2C and Supplemental Video 3). Since YopJ is a type III secretion protein that triggers cell death only upon its active injection by bacteria into host cytosol (20), the lysis of neighboring macrophages must have been preceded by their infection by live bacteria released from the originally infected cell. We also incubated Kim5-infected cells with uninfected cells and quantitated the rate of cell lysis over a 20-hour period by LDH assay. We detected lysis of only $10 \%$ of total cells by 12 hours, which presumably were the originally infected cells; however, the remaining cells lost their viability by 20 hours 
(Figure 2D). As expected, no detectable cell death was observed during a similar experiment with $\Delta y o p J$ bacteria (Figure 2D). These observations indicate that the lysis of the primary infected cell allows infection of neighboring cells, which are also lysed subsequently after uptake of bacteria.

Finally, to demonstrate cell-to-cell bacterial spread in vivo, we infected 10,000 J774A.1 cells with either OFP-labeled Kim5 or mutant bacteria and then injected these cells into the mouse footpad. We observed significantly higher numbers of infected cells after 9 hours in the PNs of mice injected with Kim5-infected macrophages compared with mice injected with $\Delta y o p J$-infected macrophages (Figure 2E), but total number of viable bacteria was still comparable (Figure $2 \mathrm{~F}$ ). Altogether, our data show that the initial burst of bacteria in the primary DLN is due to death of infected macrophages, which results in the spread of $Y$. pestis within DLN. Mechanistically, this occurs through the $Y$. pestis virulence factor YopJ.

Virulence of YopJ depends on RIPK1-mediated programed cell death. Having identified that monocyte/ macrophage lysis is a key step in $Y$. pestis reaching new cellular targets within DLNs, we questioned whether reduced cell death would be observed in $\Delta y o p J$-infected DLNs. Even as early as 24 h.p.i., distinct signs of cell death in Kim5-infected PNs could be observed after propidium iodide staining to visualize dead cells, but this was strikingly reduced in $\Delta y o p J$-infected nodes (Figure 3A). Dead cells were quantified in single cell preparations from each of the nodes by flow cytometry, which identified the majority of the dead cells as monocyte/macrophages (Supplemental Figure 3A) and supported a significant reduction in dead cells in $\Delta y o p J$-infected compared with Kim5-infected PNs (Figure 3B). Interestingly, in spite of the cell death occurring in Kim5 Y. pestis-infected PNs, their cellularity remained comparable with that observed in $\triangle y o p J$ mutant-infected PNs (Supplemental Figure 3, B and C). There was also no appreciable difference in the infiltrated phagocytic cell subtypes between Kim5 and $\Delta y o p J$ mutant-infected PNs (Supplemental Figure 2B and Supplemental Figure 3D). These results support the association of cell death in vivo with YopJ-induced virulence.

Since deficiency of RIPK1 has previously been shown to protect against $Y$. pestis-induced cell death (12), we investigated whether RIPK1 is involved in YopJ-dependent pathogenesis. RIPK1 has a kinase-independent scaffolding activity critical for neonatal development; therefore, RIPK1 deficiency is perinatally lethal (21). However, viable knock-in mice have been described, which express a kinase-inactive mutant RIPK1 (Ripk1 ${ }^{\text {DIS8N/DI38N }}$ ) that is unable to execute cell death (16). If the YopJ-dependent cell death involves RIPK1, macrophages from Ripk1 $1^{D 138 N / D 138 N}$ mice should resist death when infected by Kim5 Y. pestis. Shown in Figure $3 \mathrm{C}$ is the viability of BM-derived macrophages (BMDMs) from WT and mutant Ripk1 $1^{D 138 N / D 138 N}$ mice following infection with Kim5 or $\Delta y o p J$ Y. pestis. Although the WT and mutant macrophages were comparably infected (Supplemental Figure 4A), Ripk1 ${ }^{\text {I38N/D138N BMDMs were }}$ resistant to $Y$. pestis-induced cell death (Figure 3C), confirming that $Y$. pestis YopJ-triggered cell death is dependent on RIPK1 kinase activity. In vivo bacterial numbers in INs relative to that in respective PNs were much lower in Ripk1 ${ }^{D 138 N / D 138 N}$ mice compared with those of WT mice (Figure 3D), suggesting that RIPK1-dependent cell death is important for the amplification of infection within primary DLNs, leading to increased spread of bacteria from the primary DLN to the secondary DLNs. Consequently, compared with WT mice, Ripk1 $1^{D 138 N / D 138 N}$ mice were significantly resistant to lethal Kim5 Y. pestis challenge (Figure 3E). It is noteworthy that Ripk1 $1^{D 138 N / D 138 N}$ mice had normal sized PNs, and the size of buboes formed following Kim5 $Y$. pestis infection was also comparable with that seen with WT mice (Supplemental Figure 4B and Supplemental Figure 1D), indicating that there was no intrinsic defect in the recruitment of immune cells in mutant mice and that RIPK1 kinase activity does not substantially influence cellular recruitment into $Y$. pestis-infected DLNs.

Since RIPK1-depdendent cell death is preventable by necrostatin-1 (Nec-1), an inhibitor of RIPK1 kinase activity (22), we investigated if this inhibitory agent could also block $Y$. pestis YopJ-triggered death of infected macrophages in vitro. We observed that Nec-1 was a potent inhibitor of Y. pestis-induced death of J774A.1 macrophages (Figure 3F) and also inhibited cell-to-cell bacterial spread in vitro (Figure 3G). Nec-1 itself neither had any direct bactericidal effects nor affected bacterial invasion (Supplemental Figure 4, C and D). We, therefore, employed Nec-1s, a metabolically stable variant of this agent that also did not have any direct antibacterial activity (Supplemental Figure 4E), to demonstrate the importance of YopJ-triggered cell death in the pathogenesis of $Y$. pestis. We found that treatment of mice with Nec-1s was sufficient to significantly protect these animals from lethal $Y$. pestis infection (Figure 3H). Taken together, YopJ triggered a RIPK1-depdendent cell death program within buboes that was essential for the virulence of $Y$. pestis. Furthermore, RIPK1 is a therapeutic target for limiting $Y$. pestis dissemination. 
A

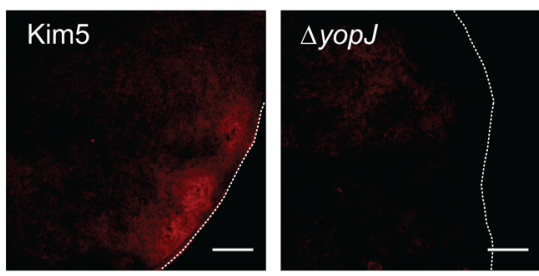

D

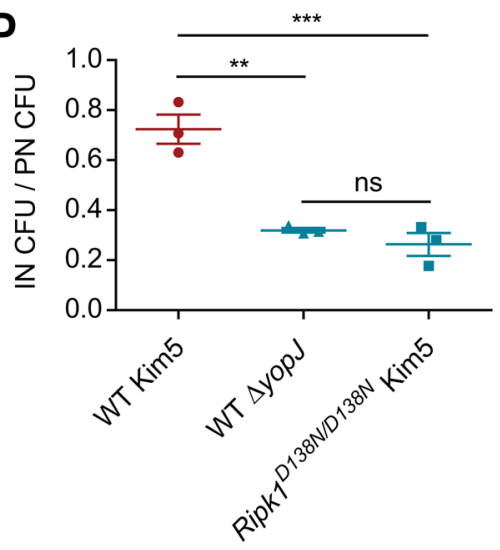

B

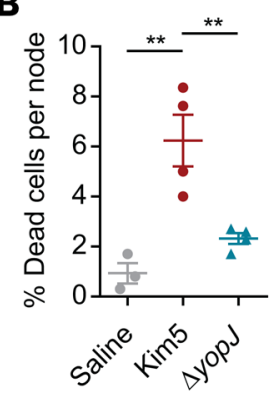

C $\quad \rightarrow \quad$ WT Kim5

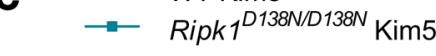

$\longrightarrow$ WT $\triangle$ yopJ

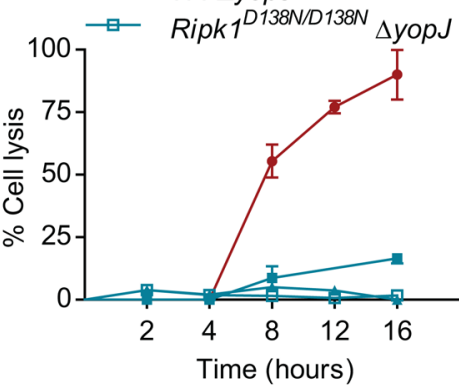

E

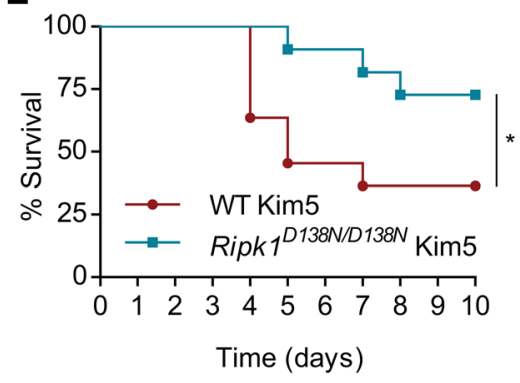

F - Media

- Nec-1

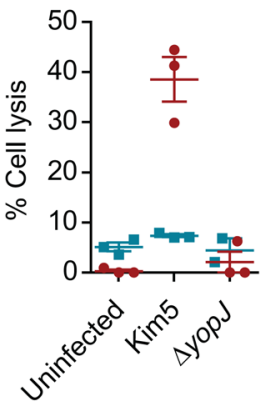

G

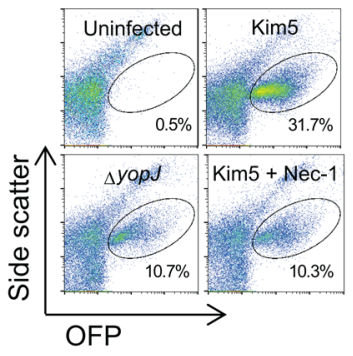

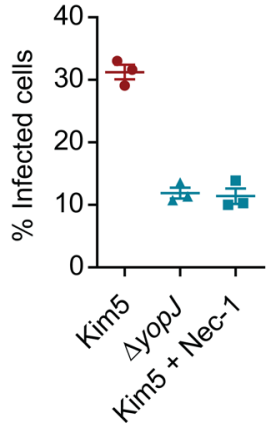

H

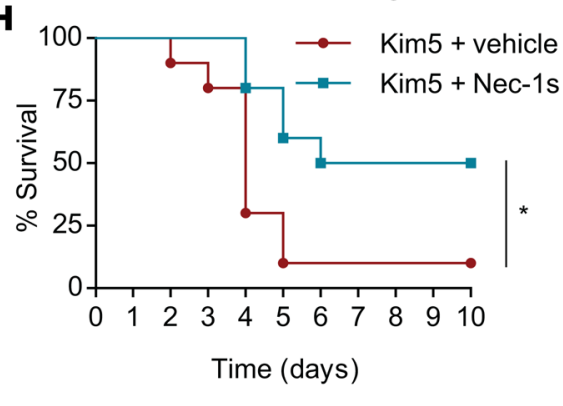

Figure 3. Virulence of YopJ depends on RIPK1-mediated programed cell death. (A) Representative images showing degree of necrosis in PNs revealed by propidium iodide staining 24 h.p.i. with Kim5 or $\Delta y o p$ J strains $(n=2)$. Scale bar $100 \mu \mathrm{m}$. (B) Quantification of dead cells in the PNs by flow cytometry ( $n=$ 3-4). Data are representative of 2 independent experiments. (C) Lysis of BMDMs isolated from WT or RipK10138N/138N mice following in vitro infection with Kim5 or $\triangle$ yopJ (MOI 10). Data are representative of 3 independent experiments. (D) Bacterial counts in INs relative to bacterial counts in PNs 24 hours after footpad infection $(n=3)$. (E) Survival of WT and RipK10138N/0138N mice following footpad challenge with Kim5 bacteria. Data are combined from 2 independent experiments, each with 5-6 mice per group. (F) Lysis of J774A.1 macrophages at 8 h.p.i. during Kim5 or $\Delta y o p /$ infections in the presence or absence of Necrostatin-1 (Nec-1, $30 \mu \mathrm{M})(n=3)$. (C) Flow cytometry plots and percentage graph showing infection of neighboring macrophages from Kim5-OFP or $\triangle y$ yop/-OFP infected macrophages in the presence or absence of Nec-1, 10 hours after initial infection $(n=3)$. Data are representative of 2 independent experiments. (H) Survival of Kim5-infected mice with or without Nec-1s treatment. Data are combined from 2 independent experiments, $n=5$ in each experiment. Significance for Kaplan-Meier curves was determined by the log-rank test. Other data were analyzed via 1-way ANOVA. ${ }^{*} P<0.05,{ }^{* *} P<0.01,{ }^{* * *} P<0.001$.

YopJ-triggered cell death is necroptotic and is activated after a distinct lag period. Programmed cell death can be defined as apoptosis, autosis, or various types of regulated necrosis such as pyroptosis and necroptosis, each triggered by distinct but often interconnected signaling programs (15). Having established the importance of $Y$. pestis-induced cell death to bacterial dissemination in vivo, we sought to further characterize the type of cell death that was induced. While RIPK1-mediated cell death programs can result in either apoptosis or necroptosis (15), our finding that bacterial exteriorization occurs from dying macrophages (Figure 2A) strongly suggests a necroptotic mode of cell death. In contrast to apoptosis, where the cellular membrane is intact, necroptosis results in membrane disruption (23), which would allow bacteria to be released extracellularly. To validate if YopJ triggers macrophage necroptosis through a RIPK1-dependent mechanism, we performed immunoprecipitation studies on BMDMs from WT or RIPK1-inactive mutant (Ripk1 1.138/D138M $)$ mice. Necroptosis is initiated when RIPK1, upon its interaction with caspase-8, becomes bound to RIPK3 to form a high molecular weight 
A

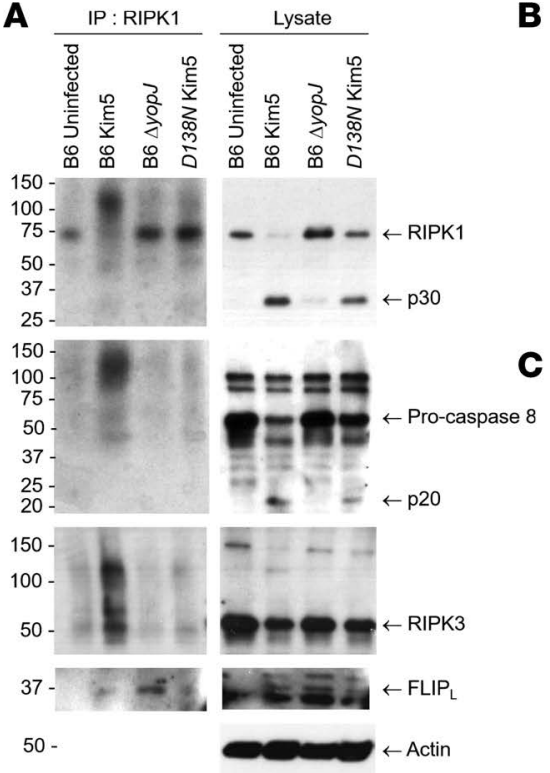

\section{C}

$\begin{array}{llllll}\text { B Time (h) } & 0 & 2 & 4 & 8 & 12\end{array}$
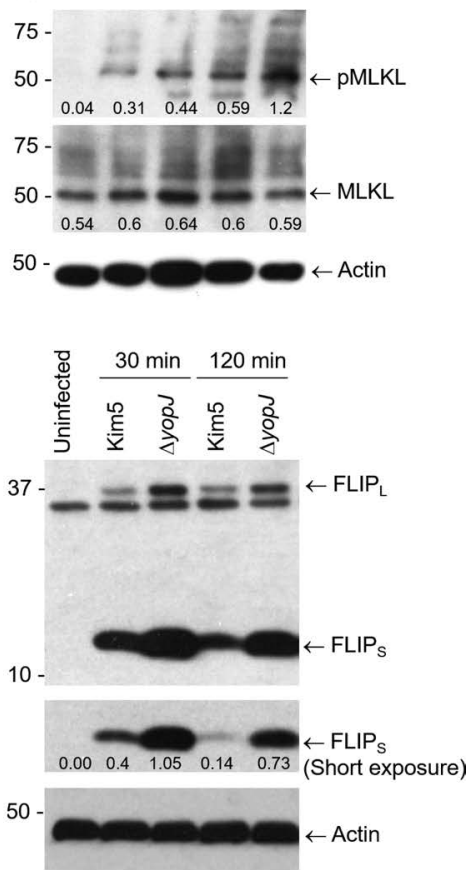

E

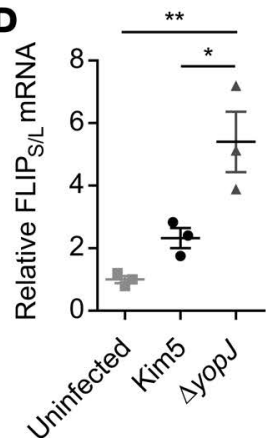

- Media

- Kim5

$\Delta$ sopJ

Figure 4. YopJ-triggered cell death is necroptotic and is activated after a distinct lag period. (A) RIPK1 immunoprecipitated from WT (B6) or RIPK1 mutant (D138N) BMDMs 3 h.p.i. and the presence of RIPK1, Caspase-8, RIPK3, and FLIP, detected by immunoblotting. Total lysates were also assayed for these proteins and $\beta$-actin. p30 and p20 indicate cleavage products of corresponding proteins. (B) Immunoblot analysis of lysates prepared from Kim5-infected BMDMs at indicated time points and probed for MLKL, phospho-MLKL, and $\beta$-actin. Intensity measurements relative to actin were shown under corresponding bands. (C) Immunoblot analysis of lysates prepared from uninfected macrophages or macrophages collected at indicated time points after infection with Kim5 or $\Delta y o p /$ strain. Immunoblots were probed with anti-FLIP S/L $_{\text {. }}$ and anti- $\beta$-actin. Band intensities relative to actin were shown for the short-exposure panel. (D) Fold change of FLIP mRNA 2 h.p.i. relative to expression in uninfected macrophages $(n=3)$. (E) Lysis of WT or Ripk $1^{0138 N / 0138 N}$ BMDMs 4 h.p.i. with $Y$. pestis Kim 5 or $\triangle y o p /$ strain in presence or absence of $10 \mu \mathrm{M}$ SAHA, a FLIP inhibitor $(n=3-4)$. Data are representative of 2 independent experiments. Data were analyzed via unpaired 2 -tailed Student's $t$ test. ${ }^{*} P<0.05,{ }^{* *} P<0.01$. See complete unedited blots in the supplemental material.

detergent-insoluble complex called necrosome $(14,18)$. Therefore, we attempted to pull down this protein complex from Y. pestis-infected BMDMs using a RIPK1-specific antibody. We found that, while the high molecular weight complex was detectable in macrophages infected with YopJ-expressing bacteria, it failed to form in the absence of YopJ (Figure 4A). As expected, there was no complex formed in macrophages derived from RIPK1 inactive mutant mice (Ripk1 $1^{\text {DI3NN/DI38M }}$ ) upon Kim5 Y. pestis infection (Figure 4A). Further confirmation of the necroptotic mode of cell death comes from the observation that MLKL protein, the executor of necroptosis (24), became progressively more phosphorylated (Figure 4B), which is the key step for the polymerization of MLKL and subsequent formation of membrane-disrupting pores. Thus, a significant portion of $Y$. pestis-infected cells, if not all, undergoes necroptosis.

We noted that macrophage lysis occurred only after a lag phase of at least 4 hours in vitro (Figure 3C), which provided sufficient time for $Y$. pestis to replicate intracellularly (Figure 2B). We, therefore, questioned 
whether the necrosome formation was delayed to provide this lag period. Since the RIPK1-mediated cell death program is highly sensitive to prosurvival factors produced by host cells, we investigated if there was a link between the delay in cell death and expression of 1 or more prosurvival factors. The Fas-associated death domain-like (FADD-like) IL-1- $\beta$ converting enzyme-like (FLICE/Caspase-8-like) inhibitory protein (FLIP) is a prosurvival factor that physically prevents the assembly of the cell death-signaling protein complex containing RIPK1, FADD, and caspase- 8 , by competitively binding 1 or more partners in the complex $(25,26)$. Indeed, we observed that FLIP was bound to the cell death-signaling complex more in the absence of YopJ, indicating that it plays a role in $Y$. pestis-induced cell death (Figure 4A).

This observation led us to hypothesize that, upon $Y$. pestis infection, any activation of the RIPK1-mediated cell death pathway in macrophages is abrogated by FLIP. YopJ interferes with this inhibitory activity, and eventually, cell death is initiated. Support for this hypothesis comes from the finding that a significant increase of expression levels of long and short isoforms of FLIP (FLIP $\mathrm{L}_{\mathrm{L}}$ and $\mathrm{FLIP}_{\mathrm{S}}$ ) was observed in $Y$. pestis-infected macrophages as early as 30 minutes after infection (Figure 4C). Amounts of FLIP $_{\mathrm{L}}$ and $\mathrm{FLIP}_{\mathrm{S}}$ in Kim5-infected cells were lower compared with $\Delta y o p J$-infected cells, and $\mathrm{FLIP}_{\mathrm{S}}$ also appeared to diminish with time (short exposure in Figure 4C). Furthermore, FLIP mRNA expression levels were lower in Kim5-infected cells compared with $\Delta y o p J$-infected cells, indicating that YopJ was influencing mRNA levels (Figure 4D). When we infected BMDMs in the presence of suberanilohydroxamic acid (SAHA), which targets FLIP for proteosomal degradation (27), we observed significant induction of early cell lysis by 4 hours, irrespective of the presence of YopJ (Figure 4E). These observations cumulatively suggest $Y$. pestis-induced cell death is delayed, at least in part, due to interference by prosurvival factors activated in macrophages, resulting in a window of time that allows for intracellular bacterial replication.

Necroptosis of infected macrophages alters local S1P gradients, promoting intranodal bacterial spread. A remarkable observation we made in the time-lapse videos tracking the spread of $Y$. pestis from infected macrophages to neighboring macrophages was the large number of surrounding cells actively moving toward the Kim5-infected macrophage (Figure 2A, top panels, and Supplemental Video 1). This directional movement of neighboring macrophages, which appeared to promote bacterial infection of a greater number of surrounding cells, was absent with $\Delta y o p J$-infected cells (Figure 2A, bottom panels, and Supplemental Video 2), suggesting that potential chemotactic signals were emanating from necroptotic cells. To verify this observation, we undertook a transwell migration assay, where we observed much greater numbers of uninfected macrophages migrating across the membrane barrier toward Kim5-infected cells compared with $\Delta y o p J$-infected cells (Supplemental Figure 5). Sphingosine 1-phosphate (S1P) has been implicated as a putative "find-me" signal secreted by dying cells to attract phagocytes to promote their clearance $(28,29)$. This chemoattractant is especially relevant here, as it is also a major chemoattractant that regulates movement of immune cells within and out of DLNs, including during $Y$. pestis infection $(4,30,31)$. To determine if S1P production might be enhanced in macrophages during $Y$. pestis infection in vitro, we assessed the expression of sphingosine kinase 1 (SphK1), the specific sphingosine-phosphorylating enzyme involved in S1P generation (32). We observed robust upregulation of Sphk1 mRNA expression in Kim5-infected cells preceding cell lysis but not in $\Delta y$ opJ-infected cells or in the presence of Nec-1 (Figure 5A). A similar effect of Nec-1 was also observed in mRNA expression of SphK2, an alternate S1P-generating enzyme (Supplemental Figure 6). Microscopic observation of infected macrophages also corroborated the RIPK1-dependent increase of SphK1 protein levels (Figure 5B), suggesting that these macrophages have the potential to produce S1P during necroptosis, resulting in the recruitment of surrounding macrophages.

If S1P production by infected and dying monocytes/macrophages also contributes to bacterial spread in infected PNs, we predicted that the deletion of S1PR1 — the corresponding receptor - would markedly impact the distribution of bacteria within PNs. To test this hypothesis, we employed a previously generated Cx3cr1-Cre S1pr flfl $^{\text {mice }}$ in which S1PR1 is deleted only in mononuclear phagocytes, and this conditional deletion does not alter the cellularity of the lymph nodes (4). We used Kim5-OFP Y. pestis for infection to investigate the distribution of bacteria in the PNs of Cx3cr1-Cre S1pr $1^{f / l f l}$ mice compared with that in PNs of littermate controls (Cx3cr1-Cre S1pr1 $\left.{ }^{+/}\right)$. We found that there was no difference between the 2 genotypes in the total number of bacteria found in the PNs (Figure 5C). However, the bacteria in the PNs of Cx3cr1-Cre S1prf ${ }^{f l f l}$ mice was limited to the peripheral region (proximal to subcapsular sinus), which is in marked contrast to the littermate controls where the dispersal of bacteria was observed throughout the node (Figure 5D). These observations suggest that, in addition to enhanced bacterial spread mediated by the necroptotic death and extracellular release of bacteria, S1P released by dying cells were attracting neighboring cells, further enhancing the spread of bacteria. 
A
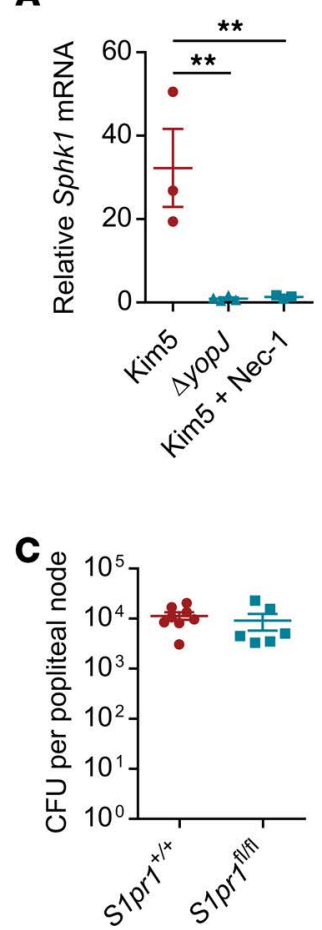

B
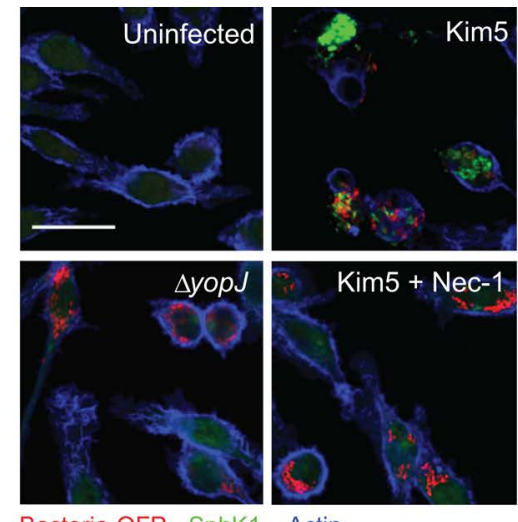

Bacteria-OFP SphK1 Actin
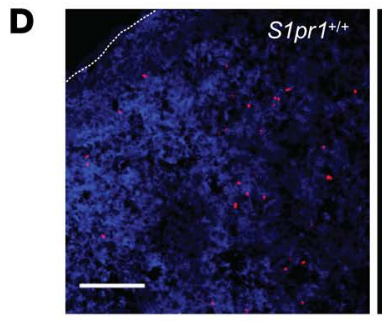

Kim5-OFP CD11b

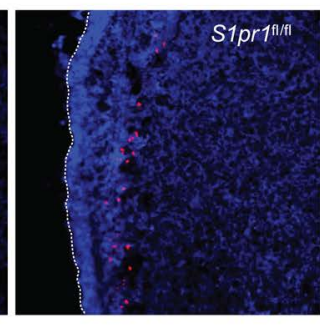

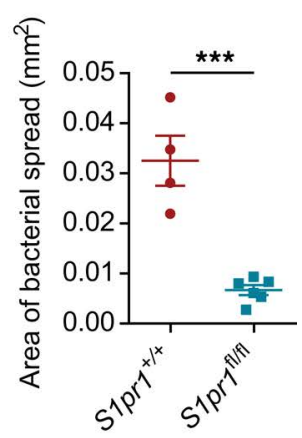

Figure 5. Necroptosis of infected macrophages alters local S1P gradients promoting intranodal bacterial spread. (A) Fold change of Sphk1 mRNA in J774A.1 macrophages at 8 h.p.i. relative to uninfected controls $(n=3)$. (B) Immunofluorescence staining for SphK1 (green) in uninfected, Kim5-OFP-infected or in $\triangle$ yop/-infected macrophages. Scale bar: 25 $\mu \mathrm{m}$. (C) Bacterial numbers in PNs from mice whose mononuclear phagocytes were S1PR1-sufficient (Cx3cr1-Cre S1pr $7^{+/+}$) or -deficient ( $\left(x 3 \mathrm{cr} 1-C r e ~ S 1 p r 7^{7 / 7 f}\right) 24$ hours following footpad infection with Kim5 Y. pestis $(n=6)$. (D) Immunofluores-

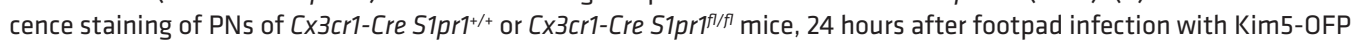
bacteria. Scale bar: $50 \mu \mathrm{m}$. Graph shows area of bacterial spread measured from the PN images $(n=4-6)$. Data were analyzed via unpaired 2-tailed Student's $t$ test or 1-way ANOVA. Data are representative of at least 2 independent experiments. ${ }^{* *} P<0.01,{ }^{* *} P<0.001$.

\section{Discussion}

Following intradermal inoculation by a flea bite, $Y$. pestis sequentially infects lymph nodes along the lymphatic system, converting each of them into swollen buboes $(5,7)$. The pathogen even co-opts the massive influx of phagocytic cells that the host has mobilized into the node to contain the pathogen by converting these cells into powerful vehicles for bacterial dispersal (4). Our studies reveal the crucial role of necroptotic cell death in amplifying the number of bacteria-bearing monocytic phagocytes within buboes and its subsequent impact on bacterial dissemination. Although extensive necrosis of buboes is routinely observed during postmortems of $Y$. pestis-infected subjects and in animal models of bubonic plaque $(5,7,8)$, its association with $Y$. pestis virulence or with any host programmed cell death pathways has gone largely unnoticed. We observed that bubo necrosis was caused by the $Y$. pestis YopJ protein, which initiates a RIPK1-dependent necroptotic form of death in infected cells. Aside from the protection offered against host factors such as complement and antibodies, the intracellular stage of $Y$. pestis is important for its dispersal through the lymphatic system, since it hitchhikes within mononuclear phagocytes to reach systemic infection (4). We also show that replication of bacteria occurs during this critical intracellular stage of infection. However, lysis of a $Y$. pestis-infected cell and exteriorization of bacteria can lead to the simultaneous infection of multiple cells and amplification of the infection in surrounding macrophages, which may then traffic to secondary nodes and spread the infection systemically. Thus, both the intra- and extracellular stages of the $Y$. pestis life cycle are critical to its virulence, and importantly, we have identified a trigger regulating the switch between these 2 stages. It is remarkable that the bubo sizes and bacterial numbers in primary buboes were comparable between Kim5- and $\Delta y o p J$-infected mice, as it indicated that, in spite of the significant cell death, neither the recruitment of immune cells into buboes nor bacterial growth was affected at this early stage 
of infection. Indeed, only the cell death-mediated difference in the number and distribution of bacteria-bearing macrophages within each node was found to be critical in determining bacterial dispersal and, ultimately, host survival.

The in vivo contribution of $Y$. pestis-mediated cell death to bacterial virulence was evident not only from studies with the YopJ mutant bacteria, but also from parallel studies using RIPK1 mutant (Ripk1 $1^{\text {D138N/D138N }}$ ) mice or RIPK1 inhibitor-treated animals infected by Kim5 Y. pestis (Figure 3, E and H). From these studies, we have identified several distinct features of YopJ-RIPK1-mediated cell death that greatly favor bacterial spread within the buboes (Figure $1 \mathrm{~F}$ ), which subsequently impacts their systemic dissemination. Firstly, the type of cell death that is triggered is necroptosis (Figure 4, A and B), which, unlike apoptosis, involves physical rupture of lysosomal and plasma membranes (23). Membrane rupture during necroptosis is more extensive than that in pyroptosis, since the cells flatten during pyroptosis but swell and burst during necroptosis (33). Disintegration of the membranes of infected cells allows for the unrestricted release of intracellular bacteria into the extracellular space, promoting expedient infection of neighboring cells (Supplemental Video 1). Unlike necrosis, which was traditionally assumed to be the type of cell death induced in $Y$. pestisinfected buboes, the process of necroptosis that we have now identified is regulated by specific signals within the host cell. Secondly, the cell death occurs after a period of lag (in excess of 4 hours), since the infected macrophages produce high levels of the prosurvival factor FLIP immediately upon infection (Figure 4C). Presumably, the macrophages attempted to block RIPK1-dependent cell death programs induced by PAMPs to prevent release of intracellular bacteria. However, through the actions of YopJ, the level of FLIP is diminished in a time-dependent fashion to allow necroptosis to occur. The downregulation of FLIP by YopJ could be via YopJ-mediated suppression of NF-kb, an inducer of FLIP (34). Interestingly, this delay in cell death in $Y$. pestis-infected macrophages could be useful for the bacteria in multiple ways. For example, this period allows for intracellular bacterial replication (Figure 2B), which would enhance the magnitude of bacterial spread when the cell membrane is disrupted. This lag period between infection and cell death would also provide sufficient time for a newly infected cell to travel from the site of infection to primary DLNs or from the primary DLN to secondary DLNs. Therefore, it is possible that Y. pestis YopJ has evolved to be less potent in triggering cell death compared with the homologous protein YopP in ancestor species $Y$. enterocolitica (35). Our findings also distinguish the role of YopJ in $Y$. pestis compared with the homologous protein in the parent species $Y$. pseudotuberculosis. In the context of $Y$. pseudotuberculosis infection, RIPK1-mediated apoptosis was beneficial to pathogen clearance by the host (36). Our results contrast with this, showing that $Y$. pestis YopJ leads to necroptosis, which is more similar to observations with lytic viruses, allowing a regulated release of the pathogen from cellular hosts. The mechanism of choosing necroptosis over apoptosis is unclear, which could be due to differences in the potency of YopJ isoforms (13), as well as differences in cell death-inducing PAMPs $(37,38)$ among various Yersinia species.

$Y$. pestis-mediated cell death attracts surrounding cells toward dying infected macrophages promoting cell-to-cell contact and fostering further infection in buboes. Massive upregulation of the chemoattractant S1P (Figure 5, A and B) was associated with the movement of cells toward Kim5-infected cells. This chemoattractant has previously been implicated as a "find-me" signal produced by dying cells to promote their elimination by phagocytes (28). Interestingly, there is growing evidence that the movement of immune cells within the node is keenly susceptible to local S1P gradients. For example, NK cells are recruited and strategically positioned in the medulla of lymph nodes in response to the enhanced extracellular presence of S1P at these sites (31). From the study of $Y$. pestis infections in mice where the S1PR1 is lacking in mononuclear phagocytes, we deduced that S1P was a key contributor to bacterial dispersal within the buboes (Figure $5 \mathrm{D}$ ), in addition to its previously described role in permitting the egress of infected cells from buboes (4). Thus, the sharp increase in the population of bacteria-bearing macrophages in Kim5-infected buboes compared with YopJ mutant-infected buboes is attributable to the complementary actions of cell lysis and secretion of S1P, which greatly facilitate infection of surrounding macrophages.

Valuable information can be gleaned by employing models of infection that closely mimic the route of bacterial progression in the body. Although it has been known for many decades that $Y$. pestis reside and even multiply in macrophages $(39,40)$ by resisting lysosomal killing $(41)$, the significance of this activity has not been fully appreciated until mouse studies tracking the infection of the skin have revealed that $Y$. pestis primarily disseminate via the lymphatics and that DLNs are hubs for the dispersal of bacteria harboring within macrophages (4). Similarly, although it has been known for more than a decade that YopJ-mediated cell death is associated with $Y$. pestis infection (10), its significance has largely remained elusive. This study 
extends our understanding of $Y$. pestis pathogenesis by revealing how YopJ directs a delayed necroptotic cell death program in order to amplify bacterial infectivity within buboes. By greatly expanding the number of bacteria-bearing cells in primary buboes, the pathogen improves its chances of successfully infecting secondary lymph nodes and reaching the circulating system in sufficient numbers to overcome host defenses.

Our study reveals that abrogating this pathogen-initiated cell death program by therapeutically targeting RIPK1 is potentially a powerful approach to combating $Y$. pestis infection, as it would prevent bacterial dissemination while still protecting the lymph nodes from destruction. This latter aspect could be important, as it will preserve the host's ability to develop protective immunity against future infections. Since this treatment does not directly target $Y$. pestis, it is also unlikely that any resistance will be developed by the pathogen. Recent findings of a series of RIPK1 inhibitors with improved potency and pharmacokinetics (42) is highly encouraging, as they could be even more effective against this pathogen.

\section{Methods}

Mice. Six- to 8-week old C57BL/6J mice were purchased from National Cancer Institute Animal Production Area or the Jackson Laboratory. Cx3cr1-Cre S1pr1 fl/fl mice generated by breeding Cx3cr1-Cre mice (the Jackson Laboratory, stock no. 025524) to S1pr $1^{\text {loxp }}$ mice (the Jackson Laboratory, stock no. 019141) (4) were maintained in the Duke University Vivarium. Ripk1 $1^{D 138 N / D 138 N}$ mice were provided by Vishva M. Dixit (Genentech, San Francisco, California, USA).

Bacterial strains. The Kim5 strain was selected for this study and regarded as WT as its growth rate was slow, due to the absence of a pigmentation $(\mathrm{pgm})$ locus associated with iron acquisition, allowing for a slower and more tractable progression of pathogenesis (43). The Kim5-OFP strain was generated previously (4), and $\Delta y o p J, \Delta y o p J$ plasmid YopJ (pYopJ), and $\Delta y o p J$-OFP strains in Kim5 background were generated in this study. All cultures of Y. pestis strains were grown in brain heart infusion (BHI) broth (Beckton Dickinson) under shaking conditions. To mimic the infectious state of bacteria that are regurgitated by fleas into the host, cultures were grown at $25^{\circ} \mathrm{C}$ (room temperature; RT) for 48-72 hours.

Cell cultures. The monocyte/macrophage cell line J774A.1 (ATCC TIB-67) was cultured in DMEM containing glucose, glutamine, and sodium pyruvate (Invitrogen) and $10 \% \mathrm{FBS}$ (HyClone) and incubated at $37^{\circ} \mathrm{C}$ with $5 \% \mathrm{CO}_{2}$ and humidification. BMDMs from WT or Ripk1 138N/D138N mice were cultured in RPMI 1640 (Invitrogen) supplemented with glutamine, 10\% FBS, and M-CSF (BioLegend) following standard protocol (44).

In vitro infections. Macrophages (J774.A1 or BMDM) were infected with bacteria in DMEM at a multiplicity of infection (MOI) of 10. Culture plates were centrifuged at $55 \mathrm{~g}$ for 2 minutes to settle down the bacteria and then incubated at $37^{\circ} \mathrm{C}$. Two h.p.i., cells were washed and treated with $10 \mu \mathrm{g} / \mathrm{ml}$ gentamicin (Invitrogen) for 1 hour and washed again, and concentration of gentamicin was reduced to $2 \mu \mathrm{g} / \mathrm{ml}$. A higher concentration of gentamicin $(50 \mu \mathrm{g} / \mathrm{ml})$ was used to treat infected cells when they were harvested to transfer into wells containing uninfected cells for cell-to-cell spread experiments.

Animal infections. For mouse infections, $1 \times 10^{5}$ CFUs of bacteria in a $20 \mu 1$ volume of sterile PBS were injected intradermally into a single rear footpad. For survival curves, $2 \times 10^{5} \mathrm{CFUs}$ of log-phase bacteria were used, and infected animals were monitored every 24 hours for death or humane endpoints.

Antibodies and reagents. For Western blot, immunofluorescence staining or flow cytometry, the following antibodies were used: rabbit anti-RIPK1 (Cell Signaling Technologies, 3493), rabbit anti-RIPK3 (Cell Signaling Technologies, 15828), rabbit anti-Caspase-8 (Cell Signaling Technologies, 4927), rabbit anti-MLKL (Cell Signaling Technologies, 37705), rabbit anti-phospho-MLKL (Ser345) (Cell Signaling Technologies, 62233), rabbit anti-FLIP ${ }_{\mathrm{S} / \mathrm{L}}$ (Santa Cruz Biotechnology Inc., 8347), Mouse anti- $\beta$-actin (MilliporeSigma, A5441), rabbit anti-SphK1 (Abcam, 71700), goat anti-rabbit IgG-HRP (Bio-Rad, 170-6515), goat anti-mouse IgG-HRP (BioRad, 170-6516), donkey anti-rabbit IgG-FITC (Jackson Immunoresearch, 711-095-152), anti-CD45 APC/Cy7 (BioLegend, 103115), anti-CD11b-PE (eBioscience, 12-0112-81), anti-CD11b-APC (eBioscience, 17-0112-81), anti-CD11b-PerCP-Cy5.5 (BD Biosciences, 561114), anti-F4/80-FITC (eBioscience, 11-4801-82), anti-Ly-6CAF700 (clone AL-21) (BD Biosciences, 561237), anti-Ly-6G-PE (clone 1A8) (BD Biosciences, 561104), antiLy-6G and -Ly-6C-PerCP Cy5.5 (clone RB6-8C5) (BD Biosciences, 552093), anti-CD3-APC (eBioscience, 17-0032-82), and anti-B220-FITC (eBioscience, 11-0452-85). Other reagents include Phalloidin-AF647 (Invitrogen, A22287), SYTO9 green fluorescent nucleic acid stain (Invitrogen, S34854), propidium iodide (Invitrogen, P21493), LIVE/DEAD fixable green dead cell stain kit (Invitrogen, L23101), Zombie Violet fixable viability kit (BioLegend, 423113), CytoTox 96 nonradioactive cytotoxicity assay kit (Promega, G1780), Nec-1 (Abcam, 141053), Nec-1s (7-Cl-O-Nec-1) (MilliporeSigma, 5.04297.0001), and SAHA (MilliporeSigma, SML0061). 
Microscopy. For tissue sectioning, isolated tissues were flash frozen in OCT compound (TissueTek) and then sectioned $(12 \mu \mathrm{m})$ on a microtome-cryostat. For propidium iodide staining, tissue sections on slides were stained for 1 minute with 1:10,000 dilution of propidium iodide, followed by washing with PBS and mounting. For all immunostaining, tissue sections were fixed with acetone for 15 minutes at $4^{\circ} \mathrm{C}$. Tissue sections were rehydrated in PBS and blocked with 1\% BSA in PBS for 20 minutes, followed by staining with antibodies, as indicated in the figure legends. A Nikon ECLIPSE TE200 confocal microscope was used to capture immunofluorescent images.

For in vitro live imaging, J774A.1 cells were grown on chambered 1.0 borosillicate coverglass system (Lab-Tek, Nunc). Additional cells were grown on 24-well plates and infected with OFP-labeled bacteria. To follow cell death, cells were prelabeled with SYTO9 green fluorescent nucleic acid stain prior to infection with unlabeled Kim5 bacteria. After gentamicin treatment for 1 hour and washing, infected cells were harvested and transferred into Lab-Tek chambers preseeded with uninfected cells. The chambers were then placed in the live cell station and maintained at $37^{\circ} \mathrm{C}$ and $5 \% \mathrm{CO}_{2}$ with humidification. Propidium iodide was applied to the culture medium to visualize cell death. Zeiss Axio Observer microscope with Metamorph software was used to capture live moments.

To image J774A.1 macrophages, cells were grown on glass coverslips and infected with OFP-labeled bacteria (MOI 10) for 30 minutes and then treated with gentamicin for 1 hour, as described previously. Cells were fixed 6 h.p.i. with 4\% PFA, blocked and permeabilized with 1\% gelatin and 0.5\% saponin in PBS, and incubated overnight with anti-SphK1 polyclonal antibody. The cells were then stained with anti-rabbit-FITC and Phalloidin-AF647 to detect SphK1 and actin (background staining), respectively, and mounted on a glass slide using Prolong Gold (Invitrogen).

Flow cytometry. Lymph nodes were isolated from mice at the time points indicated, minced in DMEM containing $10 \%$ FBS and $100 \mathrm{U} / \mathrm{ml}$ of Collagenase A (MilliporeSigma), and incubated for 30 minutes. Single cell suspensions were produced by straining the disrupted lymph nodes through a $70-\mu \mathrm{m}$ cell straining filter (BD Biosciences). Cells were then washed in PBS with 10 mM EDTA and 1\% BSA, blocked with rat/mouse normal serums and Fc receptor block (eBioscience), and stained with fluorochrome-tagged antibodies. The number of cells was counted using a hemacytometer. Dead cells were detected using LIVE/DEAD fixable Green dead cell stain kit or Zombie Violet fixable viability kit. Uninfected cells and cells stained with a single antibody were used as compensation controls. Stained cells were analyzed by BD FACSCalibur or FACSCanto IIs flow cytometer and FlowJo software version 10.1 .

Real-time PCR. RNA was purified from cells using the RNeasy Mini Kit (Qiagen), cDNA was synthesized using the iScript cDNA Synthesis kit (BioRad), and real-time PCR was performed using SYBR Green and the StepOnePlus Real-Time PCR System (Applied Biosystems), with normalization to actin expression. Primers were obtained from Integrated DNA Technologies for $\beta$-actin: $5^{\prime}$ - TGA GAG GGA AAT CGT GCG TGA CAT - 3', 5' - ACC GCT CAT TGA CGA TAG TGA TGA - 3'; FLIP: 5' -GGC TTC GCT CCC AAA ATT GAG T - 3', 5' -TTG GCT CTT TAC TTC GCC CA - 3'; SphK1: 5' - CCT GCT CAT CAA CTG CAC AC - 3', 5' - AGG TCC ACG TCA GCA ACA AA - 3'; SphK2: 5' - GTC CCT CAA TGG TGG TGG T - 3', 5' - AGC TGT TTT GAG AGC GTT GG - 3'.

CFU determination. To determine CFU in organs, infected mice were euthanized at the noted time points after infection, and organs were harvested. Organs were then homogenized in sterile water using zirconia silica beads for 2 cycles of 60 seconds each using an automatic homogenizer. Homogenates were serially diluted on BHI plates, and the colonies were counted after 48-72 hours incubation at RT. CFU in blood were determined by collecting peripheral blood at indicated time points and plating on BHI plates upon dilution in PBS. The limit of detection was 5 CFU. To determine intracellular CFUs, infected cells were treated with 10 or $50 \mu \mathrm{g} / \mathrm{ml}$ gentamicin for 1 hour, washed with PBS, and lysed by incubation with sterile water for 10 minutes. The lysates were then diluted and plated on BHI agar to count CFU.

Necrostatin treatment. For in vitro studies, cells were preincubated 2 hours prior to infection and maintained during infection with $30 \mu \mathrm{M} \mathrm{Nec}-1$. For in vivo studies, mice were treated with a metabolically stable analog of $\mathrm{Nec}-1$ with improved specificity (Nec-1s) (45). Mice were treated with $2 \mathrm{mg} / \mathrm{kg} \mathrm{Nec}-1 \mathrm{~s}$ or vehicle (6\% 2-Hydroxypropyl- $\beta$-cyclodextrin in PBS), distributed into footpad, and with i.p. injections 15 minutes before bacterial infection. Additional doses were given at $6,12,24,48$, and 72 h.p.i. (i.p. only).

LDH assay. Lactose dehydrogenase (LDH) release from $2 \times 10^{4}$ cells was determined using the CytoTox 96 Non-Radioactive Cytotoxicity Assay kit according to manufacturer's protocol. The spontaneous LDH release by cells incubated with media alone was subtracted from other data. 
Transwell migration assay. Upon bacterial infection for 2 hours and gentamicin treatment $(20 \mu \mathrm{g} / \mathrm{ml}$ for 1 hour), J774.A1 cells in a 24-well plate (Falcon) were maintained in serum-free migration buffer (DMEM with $0.1 \%$ BSA) and low concentration of gentamicin $(2 \mu \mathrm{g} / \mathrm{ml})$. A cell culture insert with 8 - $\mu$ m pore size filter (Falcon) was seeded with uninfected J774A.1 cells in serum-free migration buffer and placed on the well containing infected cells. After 14 hours incubation at $37^{\circ} \mathrm{C}$, the cells remaining in the upper chambers were removed with a cotton tip applicator. Migrated cells attaching to the lower surface of the filters were fixed with $75 \%$ ethanol for 10 minutes, allowed to dry, and then stained with $0.2 \%$ crystal violet for 10 minutes. The filters were then removed from cell culture inserts, placed on a glass slide, and mounted with a coverslip. The number of cells migrated across the filter was counted under microscope using ImageJ software (NIH) (46).

Complementation of YopJ. YopJ was amplified from Kim5 strain by PCR using primers 5' - CGC GAA TTC GAT GAT CGG ACC AAT ATC ACA AAT AA - 3' and 5' - CCC CTC GAG TTA TAC TTT GAG AAG TGT TTT ATA T - 3', inserted into a low copy expression plasmid pWSK29 (47), which was then electroporated into elecrtocompetent $\triangle y o p J$ bacteria. Colonies were screened by PCR using pWSK29-specific flanking region primers 5' - GTC ACG ACG TTG TAA AAC GAC GGC CAG - 3' and 5' - GTG GAA TTG TGA GCG GAT AAC AAT TTC - $3^{\prime}$ and confirmed by sequencing using M13 primers $\left(5^{\prime}-\right.$ GTA AAA CGA CGG CCA GT - 3' and 5' - CAG GAA ACA GCT ATG AC - 3').

Statistics. Statistical significance was determined by unpaired 2-tailed Student's $t$ tests where only 2 groups existed or by 1-way ANOVA with Tukey's post test, as appropriate. Significance for Kaplan-Meier curves was determined by the log-rank test. Differences between groups were considered significant at $P<0.05$. All error bars indicate \pm SEM. Analyses were performed using GraphPad Prism 5.0 and Microsoft Excel 2010 software.

Study approval. All animal studies were conducted with the approval of the IACUC of Duke University.

\section{Author contributions}

MA and SNA conceived the study, designed the experiments, and wrote the manuscript. MA carried out the experiments and analyzed the data. WXGA assisted with generating Cx3cr1-Cre S1pr $1^{f / f l}$ mice, and HWC contributed to microscopic studies. ALSJ and HWC provided insightful suggestions and helped edit the manuscript. MLN provided materials and guidelines to generate bacterial strains. All authors contributed to manuscript review.

\section{Acknowledgments}

We would like to thank Vishva Dixit (Genentech) for Ripk1 D138N/D138N mice, Benjamin Carlson for support with microscopy, and Michael Cook for support with flow cytometry. We also thank Abhay P.S. Rathore for critical manuscript review. This work was funded by NIH grants U01-AI082107, R01AI096305, and R56-DK095198.

Address correspondence to: Soman N. Abraham, Duke University Medical Center, Box 3020, 207 Research Drive, Jones Building Room 255, Durham, North Carolina 27710, USA. Phone: 919.684.3630; Email: soman.abraham@duke.edu.

WXGA's present address is: Division of Infectious Diseases, Cincinnati Children's Hospital Medical Center, University of Cincinnati College of Medicine, Cincinnati, Ohio, USA.

1. Junt T, Scandella E, Ludewig B. Form follows function: lymphoid tissue microarchitecture in antimicrobial immune defence. Nat Rev Immunol. 2008;8(10):764-775.

2. Bar-Haim E, Gat O, Markel G, Cohen H, Shafferman A, Velan B. Interrelationship between dendritic cell trafficking and Francisella tularensis dissemination following airway infection. PLoS Pathog. 2008;4(11):e1000211.

3. St John AL, Abraham SN. Salmonella disrupts lymph node architecture by TLR4-mediated suppression of homeostatic chemokines. Nat Med. 2009;15(11):1259-1265.

4. St John AL, et al. S1P-Dependent trafficking of intracellular yersinia pestis through lymph nodes establishes Buboes and systemic infection. Immunity. 2014;41(3):440-450.

5. Perry RD, Fetherston JD. Yersinia pestis--etiologic agent of plague. Clin Microbiol Rev. 1997;10(1):35-66.

6. Roberts L. Echoes of Ebola as plague hits Madagascar. Science. 2017;358(6362):430-431.

7. Sebbane F, Gardner D, Long D, Gowen BB, Hinnebusch BJ. Kinetics of disease progression and host response in a rat model of 
bubonic plague. Am J Pathol. 2005;166(5):1427-1439.

8. Maynard FP. Pathology and Bacteriology-The Hongkong Bubonic Plague of 1894-Report on Psorosperms in Their Relation to the Etiology of Cancer, \&c. Ind Med Gaz. 1895;30(11):451-454.

9. Monack DM, Mecsas J, Ghori N, Falkow S. Yersinia signals macrophages to undergo apoptosis and YopJ is necessary for this cell death. Proc Natl Acad Sci USA. 1997;94(19):10385-10390.

10. Zauberman A, et al. Interaction of Yersinia pestis with macrophages: limitations in YopJ-dependent apoptosis. Infect Immun 2006;74(6):3239-3250.

11. Paquette N, et al. Serine/threonine acetylation of TGF $\beta$-activated kinase (TAK1) by Yersinia pestis YopJ inhibits innate immune signaling. Proc Natl Acad Sci USA. 2012;109(31):12710-12715.

12. Weng D, et al. Caspase- 8 and RIP kinases regulate bacteria-induced innate immune responses and cell death. Proc Natl Acad Sci USA. 2014;111(20):7391-7396.

13. Zheng Y, et al. A Yersinia effector with enhanced inhibitory activity on the NF- $\mathrm{B}$ pathway activates the NLRP3/ASC/caspase-1 inflammasome in macrophages. PLoS Pathog. 2011;7(4):e1002026.

14. Vanden Berghe T, Linkermann A, Jouan-Lanhouet S, Walczak H, Vandenabeele P. Regulated necrosis: the expanding network of non-apoptotic cell death pathways. Nat Rev Mol Cell Biol. 2014;15(2):135-147.

15. Grootjans S, Vanden Berghe T, Vandenabeele P. Initiation and execution mechanisms of necroptosis: an overview. Cell Death Differ. 2017;24(7):1184-1195.

16. Newton K, et al. Activity of protein kinase RIPK3 determines whether cells die by necroptosis or apoptosis. Science. 2014;343(6177):1357-1360.

17. Oberst A, et al. Catalytic activity of the caspase-8-FLIP(L) complex inhibits RIPK3-dependent necrosis. Nature. 2011;471(7338):363-367.

18. Li J, et al. The RIP1/RIP3 necrosome forms a functional amyloid signaling complex required for programmed necrosis. Cell. 2012;150(2):339-350.

19. Wallach D, Kang TB, Dillon CP, Green DR. Programmed necrosis in inflammation: Toward identification of the effector molecules. Science. 2016;352(6281):aaf2154.

20. Cornelis GR. The Yersinia Ysc-Yop ‘type III’ weaponry. Nat Rev Mol Cell Biol. 2002;3(10):742-752.

21. Kelliher MA, Grimm S, Ishida Y, Kuo F, Stanger BZ, Leder P. The death domain kinase RIP mediates the TNF-induced NF-kappaB signal. Immunity. 1998;8(3):297-303.

22. Degterev A, et al. Identification of RIP1 kinase as a specific cellular target of necrostatins. Nat Chem Biol. 2008;4(5):313-321.

23. Vandenabeele P, Galluzzi L, Vanden Berghe T, Kroemer G. Molecular mechanisms of necroptosis: an ordered cellular explosion. Nat Rev Mol Cell Biol. 2010;11(10):700-714.

24. Wang H, et al. Mixed lineage kinase domain-like protein MLKL causes necrotic membrane disruption upon phosphorylation by RIP3. Mol Cell. 2014;54(1):133-146.

25. Tenev T, et al. The Ripoptosome, a signaling platform that assembles in response to genotoxic stress and loss of IAPs. Mol Cell. 2011;43(3):432-448.

26. Majkut J, et al. Differential affinity of FLIP and procaspase 8 for FADD's DED binding surfaces regulates DISC assembly. Nat Commun. 2014;5:3350.

27. Crawford N, et al. SAHA overcomes FLIP-mediated inhibition of SMAC mimetic-induced apoptosis in mesothelioma. Cell Death Dis. 2013;4:e733.

28. Gude DR, et al. Apoptosis induces expression of sphingosine kinase 1 to release sphingosine-1-phosphate as a "come-and-getme" signal. FASEB J. 2008;22(8):2629-2638.

29. Medina CB, Ravichandran KS. Do not let death do us part: 'find-me' signals in communication between dying cells and the phagocytes. Cell Death Differ. 2016;23(6):979-989.

30. Schwab SR, Cyster JG. Finding a way out: lymphocyte egress from lymphoid organs. Nat Immunol. 2007;8(12):1295-1301.

31. Fang V, et al. Gradients of the signaling lipid S1P in lymph nodes position natural killer cells and regulate their interferon- $\gamma$ response. Nat Immunol. 2017;18(1):15-25.

32. Kawamori T, et al. Role for sphingosine kinase 1 in colon carcinogenesis. FASEB J. 2009;23(2):405-414.

33. Chen $X$, et al. Pyroptosis is driven by non-selective gasdermin-D pore and its morphology is different from MLKL channel-mediated necroptosis. Cell Res. 2016;26(9):1007-1020.

34. Micheau O, Lens S, Gaide O, Alevizopoulos K, Tschopp J. NF-kappaB signals induce the expression of c-FLIP. Mol Cell Biol. 2001;21(16):5299-5305.

35. Mamroud E. et al. The Inverse Relationship Between Cytotoxicity of Y. pestis and Its Virulence. In: Shafferman A, Ordentlich A, Velan B, eds. The Challenge of Highly Pathogenic Microorganisms. Dordrecht, Netherlands; Springer Netherlands; 2010:44-45.

36. Peterson LW, et al. RIPK1-dependent apoptosis bypasses pathogen blockade of innate signaling to promote immune defense. $J$ Exp Med. 2017;214(11):3171-3182.

37. Minnich SA, Rohde HN. A rationale for repression and/or loss of motility by pathogenic Yersinia in the mammalian host. Adv Exp Med Biol. 2007;603:298-310.

38. Skurnik M, Peippo A, Ervelä E. Characterization of the O-antigen gene clusters of Yersinia pseudotuberculosis and the cryptic O-antigen gene cluster of Yersinia pestis shows that the plague bacillus is most closely related to and has evolved from Y. pseudotuberculosis serotype O:1b. Mol Microbiol. 2000;37(2):316-330.

39. Janssen WA, Surgalla MJ. Plague bacillus: survival within host phagocytes. Science. 1969;163(3870):950-952.

40. Straley SC, Harmon PA. Yersinia pestis grows within phagolysosomes in mouse peritoneal macrophages. Infect Immun. 1984;45(3):655-659.

41. Pujol C, et al. Yersinia pestis can reside in autophagosomes and avoid xenophagy in murine macrophages by preventing vacuole acidification. Infect Immun. 2009;77(6):2251-2261.

42. Harris PA, et al. Discovery of a First-in-Class Receptor Interacting Protein 1 (RIP1) Kinase Specific Clinical Candidate (GSK2982772) for the Treatment of Inflammatory Diseases. J Med Chem. 2017;60(4):1247-1261.

43. Brubaker RR, Beesley ED, Surgalla MJ. Pasteurella pestis: Role of Pesticin I and Iron in Experimental Plague. Science. 
1965;149(3682):422-424.

44. Zhang X, Goncalves R, Mosser DM. The isolation and characterization of murine macrophages. Curr Protoc Immunol. 2008; Chapter 14:Unit 14.1.

45. Degterev A, Maki JL, Yuan J. Activity and specificity of necrostatin-1, small-molecule inhibitor of RIP1 kinase. Cell Death Differ. 2013;20(2):366.

46. Justus CR, Leffler N, Ruiz-Echevarria M, Yang LV. In vitro cell migration and invasion assays. J Vis Exp. 2014;(88):51046.

47. Wang RF, Kushner SR. Construction of versatile low-copy-number vectors for cloning, sequencing and gene expression in Escherichia coli. Gene. 1991;100:195-199. 